PROCEEDINGS OF THE

AMERICAN MATHEMATICAL SOCIETY

Volume 128, Number 5, Pages 1563-1566

S 0002-9939(99)05163-1

Article electronically published on October 6, 1999

\title{
ON COVERING TRANSLATIONS AND HOMEOTOPY GROUPS OF CONTRACTIBLE OPEN N-MANIFOLDS
}

\author{
ROBERT MYERS
}

(Communicated by Ronald A. Fintushel)

\begin{abstract}
This paper gives a new proof of a result of Geoghegan and Mihalik which states that whenever a contractible open $n$-manifold $W$ which is not homeomorphic to $\mathbf{R}^{n}$ is a covering space of an $n$-manifold $M$ and either $n \geq 4$ or $n=3$ and $W$ is irreducible, then the group of covering translations injects into the homeotopy group of $W$.
\end{abstract}

\section{INTRODUCTION}

The homeotopy group $\mathcal{H}(W)$ of a manifold $W$ is the group of homeomorphisms Homeo $(W)$ of $W$ modulo the normal subgroup consisting of those homeomorphisms which are isotopic to the identity. Given a subgroup $G$ of $\operatorname{Homeo}(W)$ we denote by $\rho: G \rightarrow \mathcal{H}(W)$ the homomorphism which takes an element of $G$ to its isotopy class. The following theorem is a consequence of results of Geoghegan and Mihalik in [7].

Theorem (Geoghegan-Mihalik). Let $W$ be a contractible open $n$-manifold which is not homeomorphic to $\mathbf{R}^{n}$. Assume that either $n \geq 4$ or $n=3$ and $W$ is irreducible. Suppose $W$ is a covering space of an $n$-manifold $M$ and $G \cong \pi_{1}(M)$ is the group of covering translations. Then $\rho: G \rightarrow \mathcal{H}(W)$ is one-to-one.

The 3-dimensional case of this result was used by the author in [13 to give examples of contractible open 3-manifolds which non-trivially cover other non-compact 3-manifolds but cannot cover compact 3-manifolds. Geoghegan and Mihalik mention this application in their paper but do not explicitly state the $n$-dimensional theorem in the form given above. They prove much more general results in proper homotopy theory from which the theorem follows. A brief sketch of how to deduce the theorem from their results is given in section 2 .

The main result of this paper is a new, more geometric proof of this theorem which is inspired by David Wright's work in 17]. The main ingredients are the Covering Isotopy Theorem [1, 3], Wright's Orbit Lemma, and an analog of his Ratchet Lemma which we call the Isotopy Ratchet Lemma. This latter result is proven in section 3. The new proof of the theorem is given in section 4 .

Received by the editors October 17, 1997 and, in revised form, July 10, 1998.

1991 Mathematics Subject Classification. Primary 57M10; Secondary 57N10, 57N13, 57N15, 57N37, 57M60, 57S30.

Key words and phrases. Contractible open manifold, covering space, homeotopy group, mapping class group.

Research at MSRI is supported in part by NSF grant DMS-9022140.

(C)2000 American Mathematical Society 


\section{The THEOREM AS COROLlaRY}

We first summarize some proper homotopy theory, referring to [7] for further details. For our purposes we may assume that the spaces under consideration are locally finite simplicial complexes. Call a continuous function between spaces proper if pre-images of compact sets are compact; two such maps are properly homotopic if there is a homotopy between them which is a proper map. A base ray for a noncompact space $X$ is a proper map $\omega:[0, \infty) \rightarrow X$. The space is strongly connected at $\infty$ if any two base rays are properly homotopic. An exhaustion for $X$ is a sequence $\left\{C_{n}\right\}_{n \geq 0}$ of compact subsets of $X$ such that $X=\bigcup_{n \geq 0} C_{n}, C_{n} \subseteq \operatorname{Int}\left(C_{n+1}\right)$, and every path component of $X-C_{n}$ has non-compact closure. Note that every connected, open PL manifold has an exhaustion. Given a base ray $\omega$ one may assume that the exhaustion has been chosen so that $\omega([n, \infty)) \subseteq X-C_{n}$ for all $n$. Inclusion and change of basepoint along $\omega$ yield an inverse sequence of groups $\left\{\pi_{1}\left(\left(X-C_{n}\right), \omega(n)\right)\right\}$ whose limit $\pi_{1}^{e}(X, \omega)$ is called the fundamental group of $X$ at $\infty$ based at $\omega$. Up to isomorphism this group is independent of the choice of exhaustion; if $X$ is strongly connected at $\infty$, it is also independent of the choice of base ray.

A space $X$ is called $\pi_{1}$-trivial at $\infty$ or $1-L C$ at $\infty$ if for every compact subset $A$ of $X$ there is a compact subset $B$ of $X$ such that $A \subseteq B$ and every loop in $X-B$ is null-homotopic in $X-A$; it is called simply connected at $\infty$ if, in addition, $B$ can be chosen so that $X-B$ is connected. Note that for a one-ended space (such as a contractible space) these conditions are equivalent. Moreover $X$ is simply connected at $\infty$ if and only if it is strongly connected at $\infty$ and $\pi_{1}^{e}(X)$ is trivial.

Suppose $p: X \rightarrow X^{\prime}$ is a regular covering map with infinite cyclic group of covering translations $\langle g\rangle$ generated by a homeomorphism $g$. We assume $X^{\prime}$ to be a locally finite simplicial complex. By Theorem 3.1 of Geoghegan and Mihalik [7] if $X^{\prime}$ is non-compact and $g$ is properly homotopic to the identity, then $X$ is strongly connected at $\infty$; moreover if, in addition, $X$ is simply connected, then $\pi_{1}^{e}(X)$ is trivial, and so $X$ is simply connected at $\infty$.

Now suppose that $W$ is a contractible open $n$-manifold, $n \geq 3$, which is a covering space of an $n$-manifold $M$. Consider a non-trivial cyclic subgroup $\langle g\rangle$ of the group of covering translations; let $W^{\prime}$ be the quotient space $W /\langle g\rangle$. Then $W^{\prime}$ is an $n$-manifold and so has the homotopy type of a finite dimensional, aspherical CWcomplex [11. Thus $\langle g\rangle$ must be infinite (see e.g. 8, Lemma 9.4]), and so $W^{\prime}$ has the homotopy type of a circle and is therefore non-compact. It follows that $W^{\prime}$ has a PL structure (in fact a smooth structure) [12, 5, 9], and thus so does $W$; the elements of $\langle g\rangle$ then preserve this structure. If $g$ is isotopic to the identity, then by the above discussion $W$ is simply connected at $\infty$. But a contractible open $n$-manifold which is simply connected at $\infty$ is homeomorphic to $\mathbf{R}^{n}$ if either $n \geq 4$ [15, 4] or $n=3$ and it is irreducible [2, 16]. (The last two references use the nominally stronger version of simple connectivity at $\infty$ which requires that $X-B$ be simply connected; for 3-manifolds the loop theorem implies that this is equivalent to our definition.)

\section{The isotopy RATChet LEMma}

Lemma 1 (Isotopy Ratchet Lemma). Let $W$ be an open n-manifold and $g: W \rightarrow$ $W$ a homeomorphism which is isotopic to the identity. Suppose $A$ is a compact

subset of $W$. Then there is a compact subset $C$ of $W$ containing A such that for 
all $i, j \in \mathbf{Z}$ a loop $\beta$ in $W-\bigcup_{m=-\infty}^{\infty} g^{m}(C)$ is null-homotopic in $W-g^{i}(A)$ if and only if it is null-homotopic in $W-g^{j}(A)$.

Proof. Let $g_{t}$ be an isotopy with $g_{1}=g$ and $g_{0}$ equal to the identity. Let $C$ be a compact set whose interior contains $\bigcup_{t \in[0,1]} g_{t}(A)$. By the Covering Isotopy Theorem [1, 3] there is an isotopy $h_{t}$ such that $h_{t}(x)=g_{t}(x)$ for all $x \in A$ and $t \in[0,1], h_{t}(x)=x$ for all $x \in W-C$ and $t \in[0,1]$, and $h_{0}$ is the identity.

It suffices to prove the case $j=i+1$. Let $k_{t}=g^{i} \circ h_{t} \circ g^{-i}$. Then $k_{1}\left(g^{i}(A)\right)=$ $g^{i+1}(A), k_{t}(x)=x$ for all $x \in W-g^{i}(C)$, and $k_{0}$ is the identity.

Let $\beta$ be a loop in $W-\bigcup_{m=-\infty}^{\infty} g^{m}(C)$. Assume $\beta$ is null-homotopic in $W-g^{i}(A)$. Let $f: D \rightarrow W-g^{i}(A)$ be a map of the disk with $f(\partial D)=\beta$. Let $f^{\prime}=k_{1} \circ f$. Then $f^{\prime}(\partial D)=k_{1}(f(\partial D))=k_{1}(\beta)=\beta$, and $f^{\prime}(D) \cap g^{i+1}(A)=k_{1}(f(D)) \cap g^{i+1}(A)=$ $k_{1}(f(D)) \cap k_{1}\left(g^{i}(A)\right)=k_{1}\left(f(D) \cap g^{i}(A)\right)=k_{1}(\emptyset)=\emptyset$. Thus $\beta$ is null-homotopic in $W-g^{i+1}(A)$. A similar argument, replacing $k_{1}$ by $k_{1}^{-1}$, establishes the converse.

\section{THE NEW PROOF OF THE THEOREM}

Suppose $\Gamma$ is a group acting on an $n$-manifold $W$. It acts totally discontinuously [6] if for every compact subset $K$ of $W$ one has that $g(K) \cap K=\emptyset$ for all but finitely many elements $g$ of $\Gamma$. It acts without fixed points if the only element of $\Gamma$ fixing a point is the identity. Let $p: W \rightarrow W^{\prime}$ be the projection to the quotient space $W^{\prime}$ of the action. Then $\Gamma$ acts totally discontinuously and without fixed points on $W$ if and only if $p$ is a regular covering map with group of covering translations $\Gamma$ and $W^{\prime}$ is an $n$-manifold. (See [10].)

Lemma 2 (Orbit Lemma (Wright)). Let $W$ be a contractible open $n$-manifold, $n \geq$ 3 , and $g: W \rightarrow W$ a non-trivial homeomorphism such that the group $\langle g\rangle$ of homeomorphisms generated by $g$ acts totally discontinuously and without fixed points on $W$. Given compact subsets $A$ and $C$ of $W$, there is a compact subset $B$ of $W$ containing $A$ such that every loop $\alpha$ in $W-B$ is homotopic in $W-A$ to a loop in $W-\bigcup_{m=-\infty}^{\infty} g^{m}(C)$.

Proof. This is Lemma 4.1 of [17. For a somewhat shorter proof of the case when $W$ is an irreducible 3-manifold see [14].

Proof of the theorem. As noted in section 2 it suffices to show that $W$ is $\pi_{1}$-trivial at $\infty$. Given a compact subset $A$ of $W$ and a non-trivial covering translation $g$ which is isotopic to the identity we let $C$ be as in the Isotopy Ratchet Lemma and then let $B$ be as in the Orbit Lemma. Given a loop $\alpha$ in $W-B$ we apply the Orbit Lemma to homotop it in $W-A$ to a loop $\beta$ in $W-\bigcup_{m=-\infty}^{\infty} g^{m}(C)$.

Since $W$ is simply connected, $\beta$ is null-homotopic in $W$. Since $g$ is totally discontinuous $\beta$ is null-homotopic in $W-g^{i}(A)$ for some $i$, and hence by the Isotopy Ratchet Lemma it is null-homotopic in $W-A$.

\section{REFERENCES}

1. A. V. Chernavskii, Local contractibility of the group of homeomorphisms of a manifold, Math. USSR-Sb., 8 (1969), 287-333.

2. C. H. Edwards, Open 3-manifolds which are simply connected at infinity, Proc. Amer. Math. Soc. 14 (1963), 391-395. MR 27:732

3. R. D. Edwards and R. C. Kirby, Deformations of spaces of imbeddings, Ann. Math. (2) 93 (1971), 63-88. MR 44:1032 
4. M. H. Freedman, The topology of four-dimensional manifolds, J. Differential Geom. 17 (1982), 357-453. MR 84b:57006

5. M. H. Freedman and F. Quinn, Topology of 4-manifolds. Princeton Mathematical Series 39, Princeton University Press, Princeton, NJ, 1990. MR 94b:57021

6. M. Freedman and R. Skora, Strange actions of groups on spheres, J. Differential Geometry 25 (1987), 75-98. MR 88a:57074

7. R. Geoghegan and M. L. Mihalik, The fundamental group at infinity, Topology 35 (1996), 655-669. MR 97h:57002

8. J. Hempel, 3-Manifolds, Ann. of Math. Studies, No. 86, Princeton, 1976. MR 54:3702

9. R. C. Kirby and L. C. Siebenmann, Foundational essays on topological manifolds, smoothings, and triangulations. With notes by John Milnor and Michael Atiyah. Annals of Mathematics Studies, No. 88. Princeton University Press, Princeton, N.J.; University of Tokyo Press, Tokyo, 1977. MR 58:31082

10. W. Massey, Algebraic Topology: An Introduction, Graduate Texts in Mathematics No. 56, Springer-Verlag, 1977. MR 35:2271

11. J. Milnor, On spaces having the homotopy type of a $C W$-complex, Trans. Amer. Math. Soc. 90 (1959), 272-280. MR 20:6700

12. E. E. Moise, Affine structures in 3-manifolds. V. The triangulation theorem and Hauptvermutung. Ann. of Math. (2) 56, (1952), 96-114. MR 14:72d

13. R. Myers, Contractible open 3-manifolds which non-trivially cover only non-compact 3manifolds, Topology 38, (1999), 85-94. CMP 98:17

14. R. Myers, Contractible open 3-manifolds with free covering translation groups, Topology Appl., to appear.

15. L. C. Siebenmann, On detecting Euclidean space homotopically among topological manifolds, Invent. Math. 6 (1968), 245-261. MR 38:6601

16. C. T. C. Wall, Open 3-manifolds which are 1-connected at infinity, Quart. J. Math. Oxford Ser. (2) 16 (1965), 263-268. MR 31:6218

17. D. G. Wright, Contractible open manifolds which are not covering spaces, Topology 31 (1992), 281-291. MR 93f:57004

Department of Mathematics, Oklahoma State University, Stillwater, Oklahoma 74078

E-mail address: myersr@math.okstate.edu 